\title{
Rotational Dynamics of an Amphidynamic Zirconium Metal- Organic Framework Determined by Dielectric Spectroscopy
}

Zhiyu Liu, ${ }^{1}$ Yangyang Wang, ${ }^{2}$ Miguel A. Garcia-Garibay ${ }^{* 1}$

${ }^{1}$ Department of Chemistry and Biochemistry, University of California, Los Angeles, California 90095, United States

${ }^{2}$ Center for Nanophase Materials Sciences, Oak Ridge National Laboratory, Oak Ridge, Tennessee 37831, United States

mgg@chem.ucla.edu

Table of Contents

1. Synthesis of ligand and MOFs

2. Crystal structure and SEM image of dried PIZOF-2F crystals

3. PXRD pattern of dried PIZOF-2F crystals

4. Experimental dielectric constant of PIZOF-2F

5. Variable temperature $1 \mathrm{H}-13 \mathrm{C} \mathrm{CP} / \mathrm{MAS}$ spectra of PIZOF-2F

6. Cole-Cole fitting parameters

7. Calculation of energy barrier by shifting factor

8. DSC data of PIZOF-2F 


\section{Synthesis of ligand and MOFs}

Synthesis of linker: 4,4'-((2,3-difluoro-1,4-phenylene)bis(ethyne-2,1-diyl))dibenzoic acid

$4 \mathrm{~mL}$ THF and $4 \mathrm{~mL}$ of triethylamine was mixed and then purged with argon for 20 min and added to a sealed tube in argon atmosphere. Then 1,4-diiodo-2,3-difluorobenzene (200 mg), methyl 4-ethynylbenzoate (262 $\mathrm{mg})$, palladium(II) chloride triphenylphosphine $(7 \mathrm{mg})$ and copper iodide $(28 \mathrm{mg}$ ) was added to the sealed tube and the purging continued for another 10 minutes. The sealed tube was then heated to $80{ }^{\circ} \mathrm{C}$ overnight. After it cooled down to room temperature, $10 \mathrm{~mL}$ of dichloromethane was added. The reaction mixture was filtered through celite and concentrated under vacuum. The crude product was purified by column chromatography with 8:2 DCM: acetone. Yield: $64 \% 152 \mathrm{mg} .{ }^{19} \mathrm{~F}$ decoupled ${ }^{1} \mathrm{H} \mathrm{NMR}\left(300 \mathrm{MHz}, \mathrm{CDCl} \mathrm{N}_{3}\right)$ $\delta 8.66(\mathrm{~m}, 4 \mathrm{H}), 7.42(\mathrm{~m}, 4 \mathrm{H}), 7.30(\mathrm{~s}, 2 \mathrm{H}), 2.16(\mathrm{~s}, 6 \mathrm{H}) .{ }^{19} \mathrm{~F}$ NMR $(282 \mathrm{MHz}) \delta-133(\mathrm{~s}, 2 \mathrm{~F})$.

$100 \mathrm{mg}$ of dibenzoate was added to $100 \mathrm{~mL}$ of THF in a $500 \mathrm{~mL}$ flask. Then $100 \mathrm{~mL}$ aqueous solution of $\mathrm{NaOH}$ was added to the flask. The mixture was refluxed at $70^{\circ} \mathrm{C}$ overnight. Dilute $\mathrm{HCl}$ was slowly added to the mixture to precipitate the dicarboxylic acid. After filtration and drying, $85 \mathrm{mg}$ product $(91 \%)$ was collected. . $1 \mathrm{H}$ NMR(500 MHz, DMSO) $\delta 8.00(\mathrm{dd}, 4 \mathrm{H}), 7.72(\mathrm{dd}$, $4 \mathrm{H}), 7.55(\mathrm{~m}, 2 \mathrm{H}) .13 \mathrm{C}$ NMR $\delta 166.80,151.4,149.21,131.97,129.85,128.74,125.28,96.70,83.55$.

Synthesis of MOFs: In a 3 dram Teflon capped vial $15 \mathrm{mg} \mathrm{ZrCl} 4$ and $2 \mathrm{~mL}$ DMF were added. After dissolution, $12 \mathrm{mg}$ of linker and $0.5 \mathrm{~mL}$ of acetic acid was added. The vial was sonicated for $10 \mathrm{~min}$ and placed in oven preheated to $120^{\circ} \mathrm{C}$ for 24 hours. Solvent exchange was conducted with fresh DMF and acetone. Crystals in acetone was then dried under vacuum at $120{ }^{\circ} \mathrm{C}$ for 12 hours. 


\section{Crystal structure and SEM image of dried PIZOF-2F crystals}

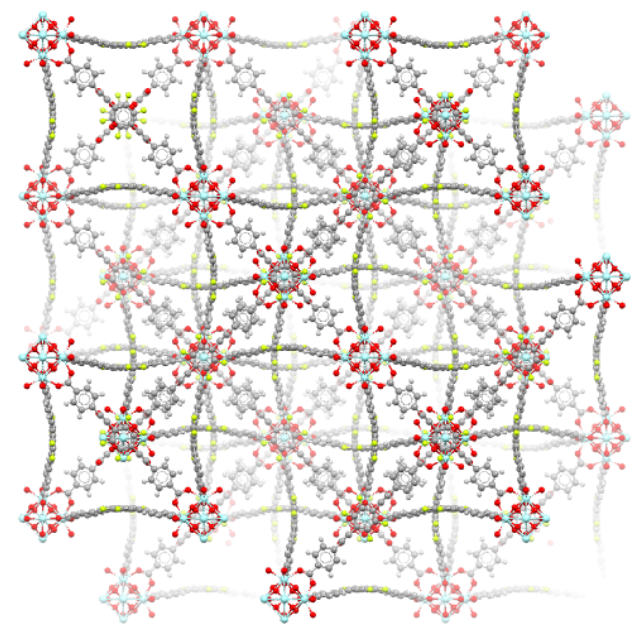

Figure S1. Crystal structure of PIZOF-2. H, light grey; C, grey; O, red; F, yellow; Zr, cyan. Single crystal X-ray diffraction data acquired at $180 \mathrm{~K}$ and $298 \mathrm{~K}$ are available in Supporting Information. 


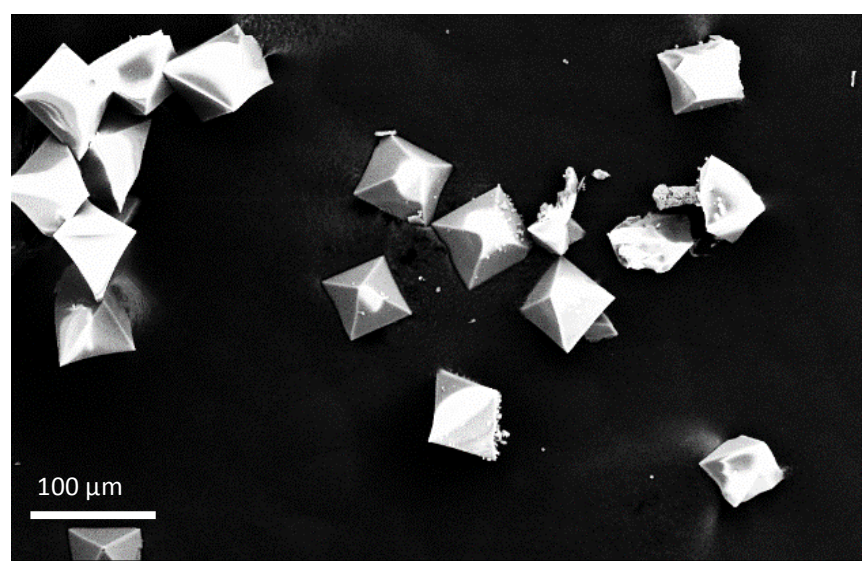

Figure S2. SEM of dried 2F-MOF crystals at $12 \mathrm{kV}$. 


\section{PXRD pattern of PIZOF-2F crystals}

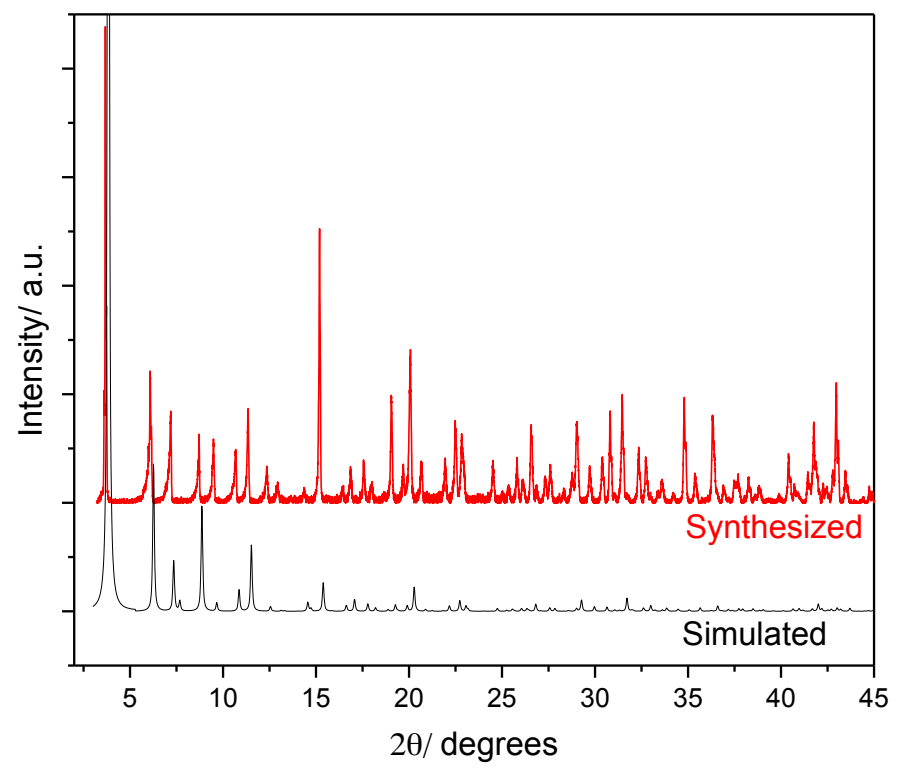

Figure S3. Power X-Ray Diffraction (PXRD) of PIZOF-2F crystals. PXRD was obtained by a Panalytical X'Pert Pro X-ray Powder Diffractometer with $\mathrm{Cu} \mathrm{K} \alpha$ radiation $(1.5406 \AA)$. 


\section{Experimental dielectric constant of PIZOF-2F}
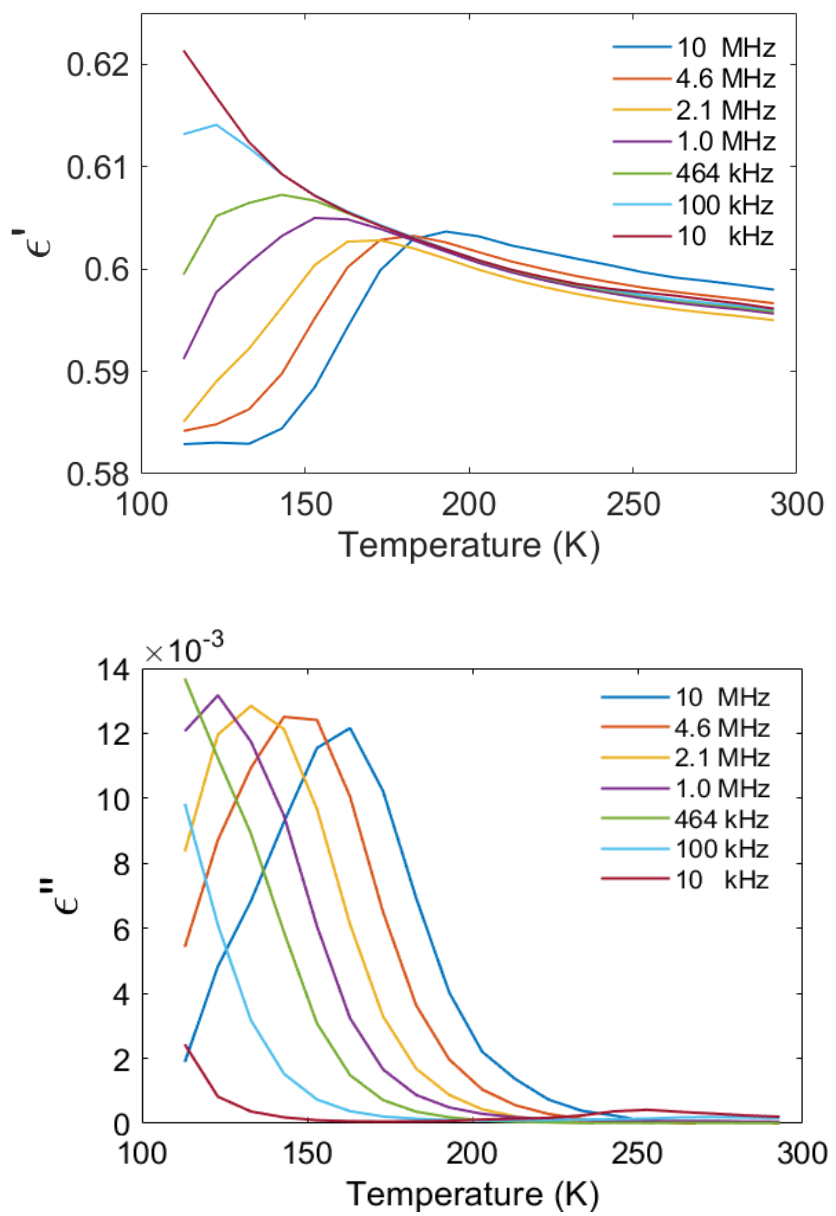

Figure S4. Experimental real (top) and imaginary (bottom) dielectric constant plot of PIZOF-2F at various frequencies.

Dielectric data was acquired with Novocontrol broadband dielectric spectrometer with Alpha-A analyzer and QUATRO cryosystem. The dried MOF crystalline powder was loaded in Teflon ring spacer sandwiched in double plate capacitor. Sample was in direct contact with electrode. Blank is measured with same setup but no MOFs loading. Void and interfaces in unpressed powder sample lead to the low capacity being measured. 


\section{Variable temperature 1H-13C CP/MAS spectra of PIZOF-2F}

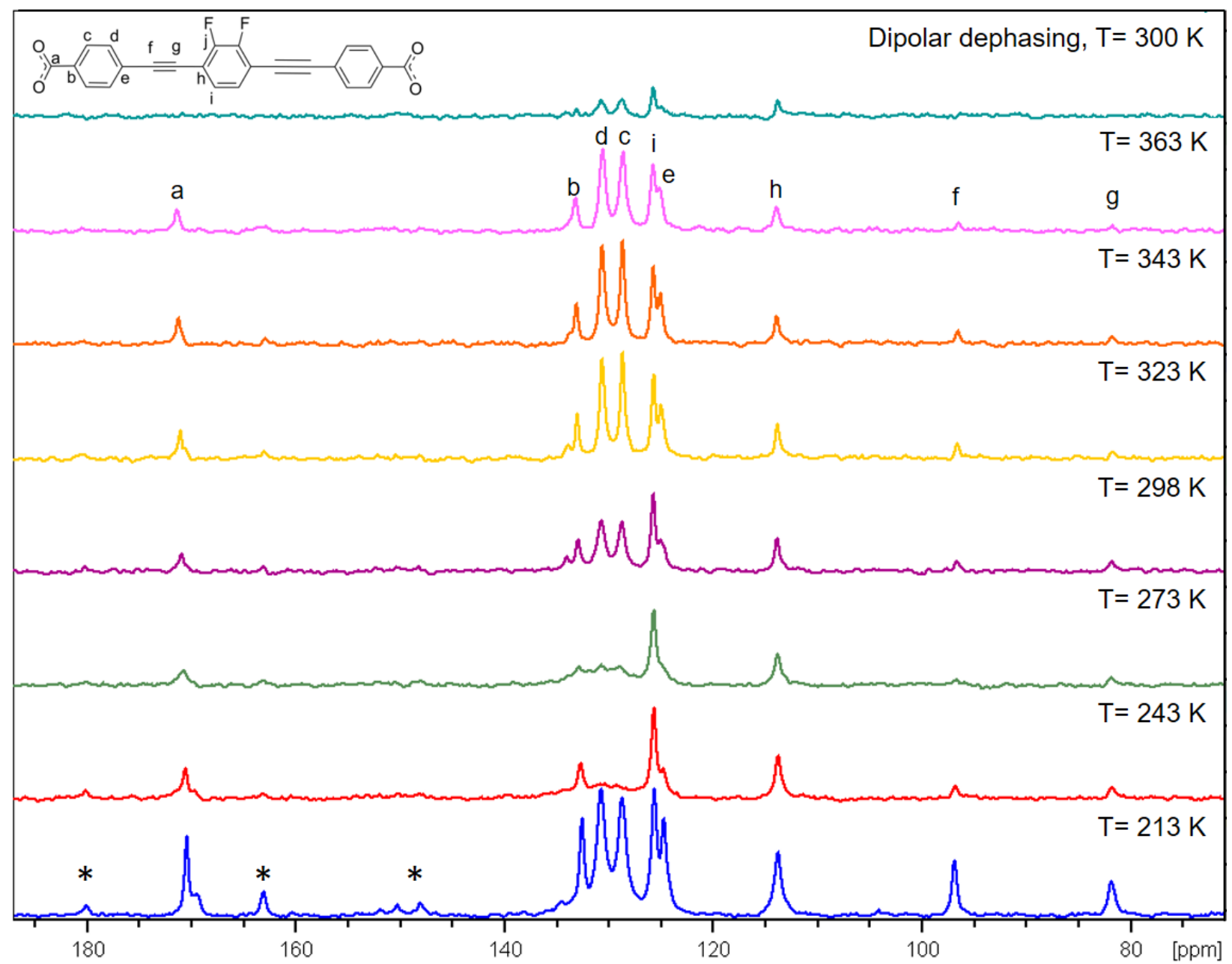

Figure S5. Variable temperature ${ }^{1} \mathrm{H}_{-}{ }^{13} \mathrm{C} \mathrm{CP} / \mathrm{MAS}$ spectra acquired by Bruker AV600SS magnet and 3.2 mm 600WB DVT HXY MAS probe. Contact time, $2 \mathrm{~ms}$. MAS rate, $10 \mathrm{kHz}$. Top spectrum was acquired with a dipolar dephasing sequence at room temperature with a dephasing delay of $50 \mu \mathrm{s}$. Disappearance and reappearance of phenylene carbons c and d near $243 \mathrm{~K}$ is due to resonance between slow dynamics of $\mathrm{c}$ and $\mathrm{d}$ and their frequencies in Hartmann-Hahn condition (c.a. $20 \mathrm{kHz}$ ). ${ }^{*}$ : Spinning side bands. 


\section{Cole-Cole fitting parameters}

\begin{tabular}{|l|l|l|l|l|}
\hline Temperature/K & $\varepsilon_{\mathrm{s}}$ & $\varepsilon_{\text {inf }}$ & $\tau / \mathrm{s}$ & $\alpha$ \\
\hline 113.15 & 0.6192 & 0.5744 & $3.40 \mathrm{E}-07$ & 0.3045 \\
\hline 123.15 & 0.6156 & 0.5728 & $1.38 \mathrm{E}-07$ & 0.2992 \\
\hline 133.15 & 0.6135 & 0.5784 & $9.74 \mathrm{E}-08$ & 0.1640 \\
\hline 143.15 & 0.6088 & 0.5812 & $7.05 \mathrm{E}-08$ & 0.0958 \\
\hline 153.15 & 0.6074 & 0.5825 & $4.13 \mathrm{E}-08$ & 0.0403 \\
\hline
\end{tabular}

Table S1. Parameters derived from Cole-Cole fitting at several temperatures. 


\section{Calculation of energy barrier from shifting factors}

Conventionally, the temperature dependency of dissipation peak frequencies is extracted from the plot and plugged in to Arrhenius equation: $\ln (f)=-E / R T+\ln (A)$, where $f$ is peak frequency, $R$ is gas constant, $E$ is activation energy, $A$ is preexponential factor. This method can still be applied to the dissipation data of PIZOF-2F by calculating temperature dependency of shifting factors though peak frequencies are not visible in the plot. After multiplying frequency data by a shifting factor $a$, slopes at higher temperatures can be brought to overlap with the slope of 113.15K (Table S2 and Figure S6). Under the assumption that lineshape remains identical, Arrhenius relations for higher temperatures become $\ln (f / a)=-E / R T+\ln (A)$. Therefore, energy barrier $E$ can still be derived from slope of $\ln (1 / a)=-E / R T+\ln (A / f)$ (Figure S7).

Table S2. Temperature dependency of shifting factor $a$

\begin{tabular}{|c|c|c|c|c|c|}
\hline Temperature/ K & 113.15 & 123.15 & 133.15 & 143.15 & 153.15 \\
\hline$a$ & 1 & 0.34 & 0.17 & 0.10 & 0.04 \\
\hline
\end{tabular}

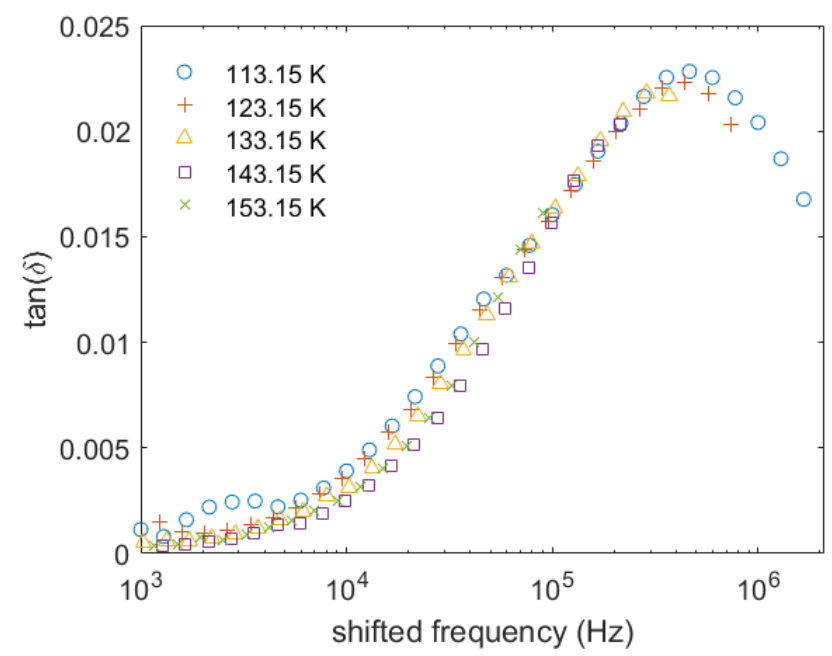

Figure S6. Dissipation factor plotted with frequency shifted by a factor of $a$ at various temperatures. 


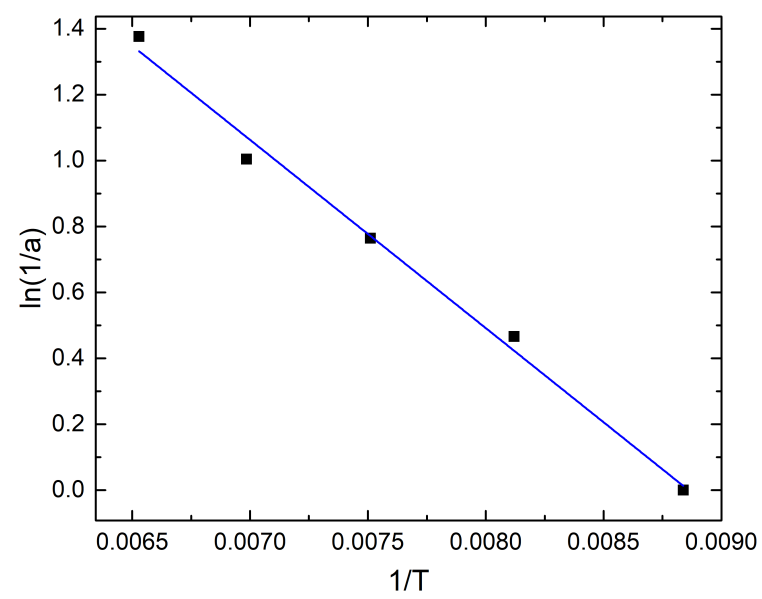

Figure S7. Linear fitting with Arrhenius equation, $\ln (1 / a)=-571 / T+5.06, R^{2}=0.989$. Energy barrier is estimated to be 2.6 kcal/mol. 


\section{DSC data of PIZOF-2F}

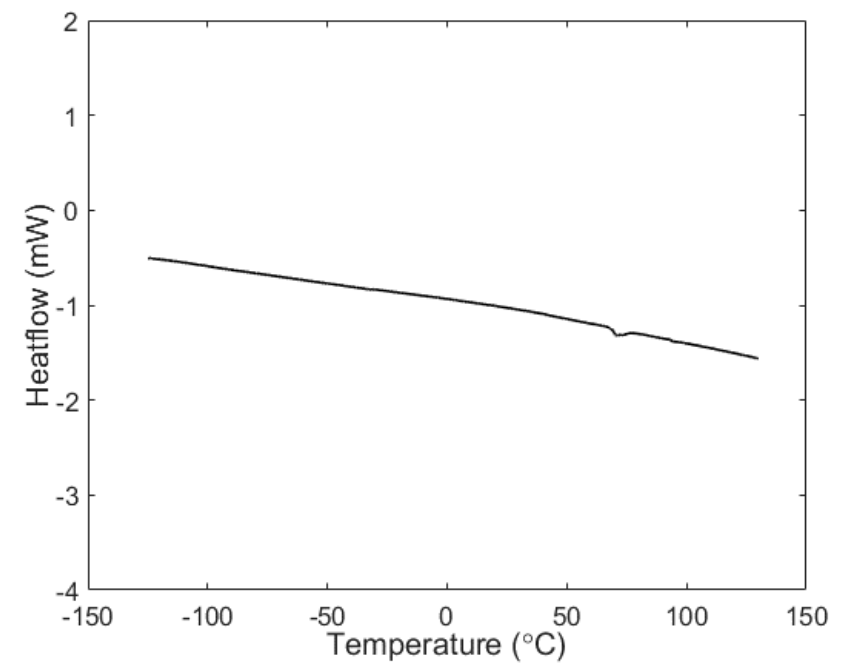

Figure S8. Differential Scanning Calorimetry (DSC) data of PIZOF-2F.

DSC data was obtained by Mettler Toledo DSC 3 system. Sample was heated from $-125{ }^{\circ} \mathrm{C}$ to $130{ }^{\circ} \mathrm{C}$ at $10{ }^{\circ} \mathrm{C} / \mathrm{min}$. No obvious phase change was observed. 